\title{
THE SOLUTION OF PROBLEMS INVOLVING THE MELTING AND FREEZING OF FINITE SLABS BY A METHOD DUE TO PORTNOV
}

\author{
by F. JACKSON \\ (Received 10th February 1964)
}

IN a recent paper (1) Portnov used a form of Poisson integral to find the exact solution for the temperature distribution in a freezing semi-infinite slab occupying the region $x>0$, and having an arbitrary time dependent temperature applied at the face $x=0$. Previously, Boley (2) had used a method based on Duhamel's theorem to find solutions for problems involving melting, in both finite and semi-finite regions, caused by time dependent heat fluxes. Steadystate solutions have been investigated by Landau (3), Masters (4) and others (5).

It is now shown that the integral derived by Portnov will also provide exact solutions for other important problems involving a change of phase in finite slabs. In Section 1 the form of solution and its physical significance is discussed briefly; in Section 2 the conditions required for its application to finite regions are derived. In Section 3 some examples of initial temperatures meeting the necessary conditions are given.

In Section 4 the solution of an ablation problem for a slab is presented, and in Sections 5, 6, 7 and 8 it is shown that the method may be applied to finite and semi-finite slabs, to solid and hollow spherical regions, and to the freezing of liquid regions. In Section 9 some examples are given. It is shown that the method gives results which agree with an example treated in (2).

\section{The Form of solution}

Let $X(t)$ be the position of the moving boundary separating the two phases of a melting slab $0<x<a \leqq \infty$. Then it can be shown that if $V_{1}(x, t)$ is a solution of the heat equation

$$
\frac{\partial V_{1}}{\partial t}=k_{1} \frac{\partial^{2} V_{1}}{\partial x^{2}}
$$

the change of variable $x=y X$ leads to the equation

$$
X^{2} \frac{\partial V_{1}}{\partial t}-X X^{\prime} y \frac{\partial V_{1}}{\partial y}=\frac{\partial^{2} V_{1}}{\partial y^{2}}
$$

where the prime indicates differentiation of a function with respect to its argument. Use of the two-sided Laplace transform with respect to $y$ results in an 
equation which can be solved readily, after which the inverse transform yields, as a formal solution of (1.1), the integral

$$
\begin{aligned}
V_{1}(x, t)=\pi^{-\frac{1}{2}} \int_{-\infty}^{\infty} \exp \left[\frac{-X^{2}\left(y-y^{\prime}\right)^{2}}{\theta^{2}}\right] \\
\quad\left\{\phi_{1}\left(X y^{\prime}\right) U\left(X y^{\prime}\right)+\phi_{2}\left(X y^{\prime}\right) U\left(-X y^{\prime}\right)\right\} \frac{X}{\theta} d y^{\prime} .
\end{aligned}
$$

Here $\theta=2(k t)^{\frac{1}{2}}, X=X(\theta), \phi_{1}(X y)$ and $\phi_{2}(X y)$ are arbitrary functions, and $U(x)$ is the unit step function. It can be seen that, in terms of the original variables,

$$
V_{1}(x, t)=\frac{1}{2}(\pi k t)^{-\frac{1}{2}} \int_{-\infty}^{\infty} \exp \left[\frac{-\left(x-x^{\prime}\right)^{2}}{4 k_{1} t}\right] f\left(x^{\prime}\right) d x^{\prime}
$$

where $f(x)=\phi_{1}(x) U(x)+\phi_{2}(x) U(-x)$. Thus (1.3) represents the temperature distribution in an infinite slab having an initial temperature equal to $f(x)$. Portnov derives (1.3) without using any boundary conditions and, since the integral can be shown to be a solution of (1.1) tending to $f(x)$ as $t \rightarrow 0$, this integral form of solution will be assumed to apply in each of the examples worked out below. As will be shown the method of determining the unknown functions $X, \phi_{1}$, and $\phi_{2}$ is to assume that they can be expanded as power series; then (1.3) is used in each of the boundary conditions, the resulting equations being repeatedly differentiated with respect to $\theta$ and the results evaluated at $\theta=0$. Each set of the resulting simultaneous algebraic equations may be solved for the successive coefficients of the unknown series. Once those have been found (1.3) may be put into the form

$$
\begin{aligned}
V_{1}(x, t) & =\pi^{-\frac{1}{2}} \int_{-X y / \theta}^{\infty} \exp \left(-\alpha^{2}\right) \sum_{n=0}^{\infty} \phi_{1 n}(\alpha \theta-X y)^{n} d \alpha \\
& +\pi^{-\frac{1}{2}} \int_{-\infty}^{-X y / \theta} \exp \left(-\alpha^{2}\right) \sum_{n=0}^{\infty} \phi_{2 n}(\alpha \theta+X y)^{n} d \alpha \ldots \ldots \ldots \ldots \ldots \ldots \ldots \ldots \ldots
\end{aligned}
$$

where

$$
i^{n} \operatorname{erfc}(x)=2 \pi^{-\frac{1}{2}} \int_{x}^{\infty} e^{-: i^{2}} \frac{(u-x)^{n}}{n !} d u
$$

is the $n$th iterated integral of erfc $x$ and is a tabulated function.

In order to attach a meaning to the functions $\phi_{1}$ and $\phi_{2}$ consider first an ablation problem where the region of interest is $X(t)<x<a$ and the temperature distribution within this region is assumed to be of the form (1.3). Then, as $t \rightarrow 0$ and $X(t) \rightarrow 0$, the initial temperature in the region $0<x<a$ is $\phi_{1}(X y)=\phi_{1}(x)$. Hence the function $\phi_{1}$ is known since the coefficients of its series development must correspond to those of the power series development of the initial temperature. The function $\phi_{2}(x) U(-x)$ represents a fictitious initial temperature in 
the half-plane $x<0$. Exactly the same remarks apply if (1.3) represents the temperature in the solid part, $X(t)<x<a$, of a melting slab in the region $0<x<a$. If, however, (1.3) represents the temperature in the liquid portion, $0<x<X(t)$ of the melting slab $0<x<a$, both $\phi_{1}(x)$ and $\phi_{2}(x)$ represent fictitious initial temperatures since the liquid region did not exist at $t=0$.

\section{Finite regions}

For simplicity consider the ablation of a slab, $X(t)<x<a$, melting under the application of a known time dependent flux $Q(t)$.

The temperature $V(x, t)$ within the slab is assumed to be given by (1.3). The conditions to be applied are

(i) an initial condition,

(ii) $V=T_{m}$ at $x=X(t)$, where $T_{m}$ is the temperature at which melting takes place,

(iii) a heat balance between $Q(t)$, the conduction of heat into the slab, and rate of melting at $x=X(t)$,

(iv) a condition of the'general type

$$
\begin{aligned}
& K \frac{\partial V}{\partial x}+H V=0 \\
& \text { at } x=a .
\end{aligned}
$$

There are four conditions but only three unknown functions, $\phi_{1}, \phi_{2}$ and $X$. As remarked in Section 1 the function $\phi_{1}$ is determined by (i). The problem appears to be overdetermined but it will now be shown that (iv) can be satisfied if the initial temperature is not arbitrary but is chosen in a particular manner.

Let the initial temperature $V_{0}$ be expanded in powers of $a-x$, that is

and define the function $\Psi_{1}(x)$ by

$$
V_{0}=V_{0}(a-x)=\sum_{n=0}^{\infty} V_{0 n}(a-x)^{n}
$$

Assume

$$
\Psi_{1}(x)=\sum_{n=0}^{\infty} \Psi_{1 n} x^{n}=V_{0}(a-x) .
$$

$$
\begin{gathered}
\phi_{2}(x)=\sum_{n=0}^{\infty} \phi_{2 n} x^{n} \\
X(t)=X(\theta)=\sum_{n=1}^{\infty} X_{n} \theta^{n}
\end{gathered}
$$

where $\theta=2(k t)^{\frac{1}{2}}$. Then

$$
\begin{aligned}
V(x, t)= & \frac{1}{\pi^{\frac{1}{2}}} \int_{-\infty}^{\infty} \exp \left[\frac{-X^{2}\left(y-y^{\prime}\right)^{2}}{\theta^{2}}\right]\left\{\psi_{1}\left(X y^{\prime}\right) U\left(X y^{\prime}\right)+\phi_{2}\left(X y^{\prime}\right) U\left(-X y^{\prime}\right)\right\} \frac{X}{\theta} d y^{\prime} \\
= & \frac{1}{\pi^{\frac{1}{2}}} \int_{-X y / \theta}^{\infty} \exp \left(-\alpha^{2}\right) V_{0}(a-\alpha \theta-X y) d \alpha \\
& \quad+\frac{1}{\pi^{\frac{1}{2}}} \int_{-\infty}^{-X y / \theta} \exp \left(-x^{2}\right) \dot{\phi}_{2}(\alpha \theta+X y) d \alpha \quad \ldots . .(2.6)
\end{aligned}
$$


and boundary condition (iv) at $x=a$ is

$$
\begin{aligned}
0 & =\frac{H}{\pi^{\frac{1}{2}}} \int_{-\alpha / \theta}^{\infty} e^{-\alpha^{2}} \sum_{n=0}^{\infty}(-1)^{n} V_{0 n} \alpha^{n} \theta^{n} d \alpha+\frac{2 K}{\theta \pi^{\frac{1}{2}}} \int_{-a / \theta}^{\infty} e^{-\alpha^{2}} \sum_{n=0}^{\infty}(-1)^{n} V_{0 n} \alpha^{n+1} \theta^{n} d \alpha \\
& +\frac{H}{\pi^{\frac{3}{2}}} \int_{-\infty}^{-\alpha / \theta} e^{-\alpha^{2}} \phi_{2}(a+\alpha \theta) d \alpha+\frac{2 K}{\theta \pi^{\frac{1}{2}}} \int_{-\infty}^{-a / \theta} e^{-\alpha^{2}} \phi_{2}(a+\alpha \theta) d \alpha
\end{aligned}
$$

or, since this is true for all $\theta$,

$$
\begin{aligned}
0 & =\frac{H}{\pi^{\frac{1}{2}}} \int_{-a / \theta}^{\infty} e^{-\alpha^{2}} \sum_{n=0}^{\infty}(-1)^{n} V_{0 n} \alpha^{n} \theta^{n+1} d \alpha+\frac{2 K}{\pi^{\frac{1}{2}}} \int_{-a / \theta}^{\infty} e^{-\alpha^{2}} \sum_{n=0}^{\infty}(-1)^{n} V_{0 n} \alpha^{n+1} \theta^{n} d \alpha \\
& \left.+\frac{\theta H}{\pi^{\frac{3}{2}}} \int_{-\infty}^{-a / \theta} e^{-\alpha^{2}} \phi_{2}(a+\alpha \theta) d \alpha+\frac{2 K}{\pi^{\frac{3}{2}}} \int_{-\infty}^{-a / \theta} e^{-\alpha^{2}} \phi_{2}(a+\alpha \theta) d \alpha . \quad \ldots \ldots \ldots \ldots . . . . .7\right)
\end{aligned}
$$

Equation (2.7) is trivially true for $\theta=0$. The $n$th derivative of the right hand side with respect to $\theta$ evaluated at $\theta=0$ will consist of the two integrals.

$$
\frac{(-1)^{n-1} n ! V_{0, n-1} H}{\pi^{\frac{1}{2}}} \int_{-\infty}^{\infty} \alpha^{n-1} e^{-\alpha^{2}} d \alpha+(-1)^{n} \frac{n ! 2 V_{0 n} K}{\pi^{\frac{1}{2}}} \int_{-\infty}^{\infty} x^{n+1} e^{-\alpha^{2}} d \alpha
$$

and terms containing

$$
\frac{\partial^{r}}{\partial \theta^{r}}\left[(a / \theta)^{\prime}(a / \theta)^{s} \exp -(a / \theta)^{2}\right]_{\theta=0} \quad r, s<n .
$$

Since all such terms typified by (3.4) are zero, the $n$th derivative of (2.7) evaluated at $\theta=0$ is (2.8) set equal to zero. The resulting equation is true if $n$ is even for then both integrals are odd functions of $\alpha$. If $n$ is odd the equation, after evaluating the integrals, is

$$
H V_{0, n-1}-n K V_{0, n}=0
$$

and the boundary condition is constant with respect to $\theta$, i.e. true for all $t>0$ if

$$
H V_{0,2 n}-(2 n+1) K V_{0,2 n+1}=0, \quad n=1,2,3, \ldots
$$

If $H=0$ then $V_{0,2 n+1}=0$ and $V_{0}(a-x)$ must be a series in even powers of $a-x$.

If $K=0$ then $V_{0,2 n}=0$ and $V_{0}(a-x)$ must be a series in odd powers of $a-x$.

If neither $H$ nor $K$ is zero then the relationship

$$
h V_{0,2 n}-(2 n+1) V_{0,2 n+1}=0,
$$

where $h=H / K$, must hold between the coefficients of the series expression for $V_{0}(a-x)$.

\section{Examples of initial temperatures}

In most cases of practical importance the material begins to melt only after its temperature has been raised from some previous level. Hence it will be assumed that melting begins after the material has been heated from zero, and 
when the face $x=0$ has reached the melting temperature $T_{m}$. Similarly a freezing body will be assumed to be in the process of cooling from some previous higher temperature. The following important examples have been adapted from (5) (see pages 112, 113, 122, 242, 238 and 125).

A. The slab $0<x<a$, zero temperature at $t=-t_{m}$, constant flux $Q_{0}$ into the region at $x=0$. No flow of heat at $x=a$.

$$
\begin{aligned}
V_{0}(a-x) & =\frac{Q_{0} t_{m}}{\rho c a}+\frac{Q_{0} a}{K}\left\{\frac{3(a-x)^{2}-a^{2}}{6 a^{2}}-\frac{2}{\pi^{2}} \sum_{n=1}^{\infty} \frac{(-1)^{n}}{n^{2}} \exp \left(-k n^{2} \pi^{2} t_{m} / a^{2}\right)\right. \\
\quad \times \cos n \pi(a-x) / a & \\
& =\frac{2 Q_{0}\left(k t_{m}\right)^{\frac{1}{2}}}{K} \sum_{n=0}^{\infty}\left\{i \operatorname{erfc}\left(\frac{2 n a+x}{2\left(k t_{m}\right)^{\frac{1}{2}}}\right)+i \operatorname{erfc}\left(\frac{(2 n+2) a-x}{2\left(k t_{m}\right)^{\frac{1}{2}}}\right)\right\} . \quad \ldots(3.1)
\end{aligned}
$$

B. The slab $0<x<a$, zero temperature at $t=-t_{m}$ constant flux $Q_{0}$ into the region at $x=0 . \quad x=a$ kept at zero temperature.

$$
\begin{aligned}
V_{0}(a-x)= & \frac{Q_{0}(a-x)}{K}-\frac{8 Q_{0} a}{K \pi^{2}} \sum_{n=0}^{\infty} \frac{(-1)^{n}}{(2 n+1)^{2}} \exp \left\{-k(2 n+1)^{2} \pi^{2} t_{m} / 4 a^{2}\right\} \\
& \times \sin \frac{(2 n+1) \pi(a-x)}{2 a} \\
= & \frac{2 Q_{0}\left(k t_{m}\right)^{\frac{1}{2}}}{K} \sum_{n=0}^{\infty}(-1)^{n}\left\{i \operatorname{erfc}\left(\frac{2 n a+x}{2\left(k t_{m}\right)^{\frac{1}{2}}}\right)-i \operatorname{erfc}\left(\frac{(2 n+2) a-x}{2\left(k t_{m}\right)^{\frac{1}{2}}}\right)\right\} .
\end{aligned}
$$

C. The slab $0<x<a$. Constant temperature $T_{0}>T_{m}$ at $t=-t_{m}$. Radiation at $x=0$ into a medium at zero. No flow of heat at $x=a$.

$$
V_{0}(a-x)=2 T_{0} a h \sum_{n=1}^{\infty} \frac{\cos \left\{\alpha_{n}(a-x) / a\right\} \exp \left(-\alpha_{n}^{2} k t_{m} / a^{2}\right)}{\left\{a h(a h+1)+\alpha_{n}^{2}\right\} \cos \alpha_{n}}
$$

where the $x_{n}$ are the positive roots of

$$
\alpha \tan \alpha=a h .
$$

D. The sphere $0 \leqq r<a$. Zero temperature at $t=-t_{m}$. Constant flux $Q_{0}$ at $r=a$.

$V_{0}(r)=\frac{3 Q_{0} t_{m}}{\rho c a}+\frac{Q_{0}\left(5 r^{2}-3 a^{2}\right)}{10 K a}-\frac{2 Q_{0} a^{2}}{K r} \sum_{n=1}^{\infty} \frac{\sin \left(\alpha_{n} r / a\right)}{\alpha_{n}^{2} \sin \alpha_{n}} \exp \left(-k \alpha_{n}^{2} t_{m} / a\right)$

where the $\alpha_{n}$ are the roots of

$$
\tan \alpha=\alpha .
$$

E. The sphere $0 \leqq r<a$. Constant temperature $T_{0}>T_{m}$ at $t=-t_{m}$. Radiation into a medium at zero at $r=a$.

$V_{0}(r)=\frac{2 h T_{0}}{r} \sum_{n=1}^{\infty} \exp \left(-k \alpha_{n}^{2} t_{m}\right) \frac{a^{2} \alpha_{n}^{2}+(a h-1)^{2}}{\alpha_{n}^{2}\left[a^{2} \alpha_{n}^{2}+a h(a h-1)\right]} \sin a \alpha_{n} \sin r \alpha_{n}$, 
where the $\alpha_{n}$ are the roots of

$$
a \alpha \operatorname{cost} a \alpha+a h-1=0 .
$$

F. The region $0<x<a$ with zero temperature at $t=-t_{m}$. Constant flux $Q_{0}$ into the solid at $x=0$; at $x=a$ radiation into a medium at zero.

$$
V_{0}(a-x)=\frac{Q_{0}}{K h}\left[1+h(a-x)-\sum_{n=1}^{\infty} \frac{2 a h\left(\alpha_{n}^{2}+a^{2} h^{2}\right) \cos \left(\alpha_{n} x / a\right)}{\alpha_{n}^{2}\left[a h(1+a h)+\alpha_{n}^{2}\right]} \exp \left(-k \alpha_{n}^{2} t_{m} / a^{2}\right)\right]
$$

where the $\alpha_{n}$ are the roots of

$$
\alpha \tan \alpha=a h .
$$

In each case $t_{m}$ has to be found by setting $V_{0}(a)=T_{m}$ and letting $x=0$ or $r=a$. Tables of the first six values of the various $\alpha_{n}$ are given in (5) and it can be seen that the series converge rapidly. Hence the value of $t_{m}$ can be found by trial and error.

It can be seen immediately that (3.1) and (3.3) are even functions of $a-x$ and that (3.2) is an odd function of $a-x$ thus satisfying equation (2.11) for the cases $H=0$ and $K=0$.

The use of (3.4) and (3.5) in problems involving spheres will be shown in Section 6 below.

(3.6) is now used to show that it satisfies (2.12), for

$$
\begin{aligned}
& V_{0}(a-x)= \frac{Q_{0}}{K h}[1+h(a-x) \\
&- \sum_{n=1}^{\infty} \frac{2\left(\alpha_{n}^{2}+a^{2} h^{2}\right) \exp \left(-k \alpha_{n}^{2} t_{m} / a^{2}\right)}{\alpha_{n}^{2}\left[a h(1+a h)+\alpha_{n}^{2}\right]}\left(\sin \alpha_{n}\right)\left\{\alpha_{n} \cos \left(\alpha_{n}(a-x) / a\right)\right. \\
&\left.\left.\quad+a h \sin \left(\alpha_{n}(a-x) / a\right)\right\}\right] \\
&= \frac{Q_{0}}{K h}+\frac{Q_{0}}{K}(a-x)-\frac{2 Q_{0}}{K h} \sum_{n=1}^{\infty} \frac{\left(\alpha_{n}^{2}+a^{2} h^{2}\right) \exp \left(-k \alpha_{n}^{2} t_{m} / a^{2}\right) \sin \alpha_{n}}{\alpha_{n}^{2}\left[a h(1+a h)+\alpha_{n}^{2}\right]} \\
& \times \sum_{r=0}^{\infty}(-1)^{r}\left\{\frac{\alpha_{n}^{2 r+1}(a-x)^{2 r}}{a^{2 r}(2 r) !}+\frac{h \alpha_{n}^{2 r}+1(a-x)^{2 r+1}}{a^{2 r}(2 r+1) !}\right\} \\
&= \frac{Q_{0}}{K h}\left[1-2 \sum_{n=1}^{\infty} \frac{\left.\left(\alpha_{n}^{2}+a^{2} h^{2}\right) \exp \left(-k \alpha_{n}^{2} t_{m} / a^{2}\right) \sin \alpha_{n}\right]}{\alpha_{n}\left[a h(1+a h)+\alpha_{n}^{2}\right]}\right] \\
&+\frac{Q_{0}}{K}\left[1-2 \sum_{n=1}^{\infty} \frac{\left(\alpha_{n}^{2}+a^{2} h^{2}\right) \exp \left(-k \alpha_{n}^{2} t_{m} j a^{2}\right) \sin \alpha_{n}}{\alpha_{n}\left[a h(1+a h)+\alpha_{n}^{2}\right]}\right](a-x) \\
&-\frac{2 Q_{0}}{K h} \sum_{r=1}^{\infty} \frac{(-1)^{r} S_{r}}{a^{2 r}(2 r+1) !}\left\{(2 r+1)(a-x)^{2 r}+h(a-x)^{2 r+1}\right\} \quad \ldots \ldots(3.7)
\end{aligned}
$$

where

$$
S_{r}=\sum_{n=1}^{\infty} \frac{\alpha_{n}^{2 r-1}\left(\alpha_{n}^{2}+a^{2} h^{2}\right) \exp \left(-k \alpha_{n}^{2} t_{m} / a^{2}\right) \sin \alpha_{n}}{a^{2 r}\left[a h(1+a h)+\alpha_{n}^{2}\right]}
$$


From (3.7) it can be seen that (2.12) is satisfied. The rearrangement of (3.6) is permissible since

$$
\left|\frac{V_{0,2 r+1}}{V_{0,2 r}}\right|=\frac{h}{2 r+1} \text { and }\left|\frac{V_{0,2 r}}{V_{0,2 r-1}}\right|=\frac{\alpha_{n}^{2}}{2 a^{2} r} ;
$$

thus, for fixed $n$, both ratios tend to 0 as $r \rightarrow \infty$, hence the series (3.7) is absolutely convergent and its terms may be grouped in any manner without altering the value of their sum. The radius of the circle of convergence is infinite. Hence (3.5), (3.7) and the corresponding series for $\Psi_{1}(x)$ defined in (2.3) will all represent the same function.

The absolute convergence of the power series for each $V_{0}(a-x)$ given above may easily be proved in similar fashion and hence each rearrangement into the form $\Psi_{1}(x)$ is justified.

4. The ablation of a slab, comprising the region $0<X(t)<x<a$, subject to a time-dependent flux at the moving boundary

Let

$$
V(x, t)=\frac{1}{\pi^{\frac{1}{2}}} \int_{-\infty}^{\infty} \exp \left[\frac{-X^{2}\left(y-y^{\prime}\right)^{2}}{\theta^{2}}\right]\left\{\psi_{1}\left(X y^{\prime}\right) U\left(X y^{\prime}\right)+\phi_{2}\left(X y^{\prime}\right) U\left(-X y^{\prime}\right)\right\} \frac{X}{\theta} d y^{\prime}
$$

be a solution of the heat equation in the solid region $X(\theta)<x<a$ subject to the conditions

$$
\begin{array}{cc}
V(x, 0)=V_{0}(a-x) & 0<x<a \ldots \ldots \\
V\{X(t), t\}=T_{m} & \ldots \ldots \ldots \ldots \ldots \ldots \ldots \ldots \ldots \ldots \\
Q(\theta)+K \frac{\partial V}{\partial x}=L \rho \frac{d X}{d t} & \text { at } x=X(t) \\
\frac{\partial V}{\partial x}=0 & \text { at } x=a
\end{array}
$$

where $L$ represents the latent heat of fusion. It is assumed that $\Psi_{1}, \phi_{2}$ and $X$ have series representations as defined in (2.3)-(2.5) and that $Q(\theta)$ can also be expressed by

$$
Q(\theta)=\sum_{n=0}^{\infty} Q_{n} \theta^{n}
$$

$V_{0}(a-x)$ must be an even function of $a-x$ if (4.2) and (4.5) are to be satisfied.

Condition (4.3) is

$T_{m}=\frac{1}{\pi^{\frac{1}{2}}} \int_{-X / \theta}^{\infty} \exp \left(-\alpha^{2}\right) \Psi_{1}(\alpha \theta+X) d \alpha+\frac{1}{\pi^{\frac{1}{2}}} \int_{-\infty}^{-X / \theta} \exp \left(-\alpha^{2}\right) \phi_{2}(\alpha \theta+X) d \alpha$.

As $\theta \rightarrow 0$ this becomes

$$
2 T_{m}=\Psi_{1}(0)\left(1+\operatorname{erf} X_{1}\right)+\phi_{2}(0)\left(1-\operatorname{erf} X_{1}\right)
$$


Therefore

since $\Psi_{1}(0)=T_{m}$.

$$
\phi_{20}=T_{m}
$$

Condition (4.4) is

$\theta Q(\theta)-2 k \rho L \frac{d X}{d \theta}=\frac{-2 K}{\pi^{\frac{1}{2}}}\left[\int_{-X / \theta}^{\infty} \exp \left(-\alpha^{2}\right) \psi_{1}(\alpha \theta+X) d \alpha\right.$

As $\theta \rightarrow 0$ this becomes

$$
\left.+\int_{-\infty}^{-X / \theta} \exp \left(-\alpha^{2}\right) \phi_{2}(\alpha \theta+X) d \alpha\right]
$$

and therefore

$$
2 k \rho L X_{1}=\frac{K}{\pi^{\frac{1}{2}}}\left\{\psi_{1}(0)-\phi_{2}(0)\right\}
$$

$$
X_{1}=0 .
$$

The first derivatives of (4.6) and (4.8) with respect to $\theta$ are

$$
\begin{aligned}
& 0=\frac{1}{\pi^{\frac{1}{2}}} \int_{-\gamma}^{\infty}\left(\alpha+X^{\prime}\right) \exp \left(-\alpha^{2}\right) \Psi_{1}^{\prime} d \alpha+\frac{1}{\pi^{\frac{1}{2}}} \int_{-\infty}^{-\gamma}\left(\alpha+X^{\prime}\right) \exp \left(-\alpha^{2}\right) \phi_{2}^{\prime} d \alpha \\
& +\frac{1}{\pi^{\frac{1}{2}}} \gamma^{\prime} \exp \left(-\gamma^{2}\right)\left\{\Psi_{1}(0)-\phi_{2}(0)\right\}, \\
& Q+\theta Q^{\prime}-2 k \rho L X^{\prime \prime}=\frac{-2 K}{\pi^{\frac{1}{2}}}\left[\int_{-\gamma}^{\infty} \alpha\left(\alpha+X^{\prime}\right) \exp \left(-\alpha^{2}\right) \Psi_{1}^{\prime} d \alpha\right.
\end{aligned}
$$

$$
\left.+\int_{-\infty}^{-\gamma} \alpha\left(\alpha+X^{\prime}\right) \exp \left(-\alpha^{2}\right) \phi_{2}^{\prime} d \alpha-\gamma^{\prime} \gamma \exp \left(-\gamma^{2}\right)\left\{\Psi_{1}(0)-\phi_{2}(0)\right\}\right]
$$

where $\gamma=X / \theta$.

Evaluated at $\theta=0$ the derivatives are

Hence

$$
\begin{gathered}
0=\Psi_{11}-\phi_{21} \\
Q_{0}-4 k \rho L X_{2}=-\frac{1}{2} K\left(\Psi_{11}+\phi_{21}\right) .
\end{gathered}
$$

$$
\begin{aligned}
\phi_{21} & =\Psi_{11} \\
X_{2} & =\frac{K \Psi_{11}+Q_{0}}{4 k \rho L} .
\end{aligned}
$$

In the examples given later $Q_{0}=-K \Psi_{11}$ and $X_{2}$ is zero.

The second derivatives of (4.6) and (4.8) are

$$
\begin{aligned}
0=\frac{1}{\pi^{\frac{1}{2}}} \int_{-\gamma}^{\infty}\left\{X^{\prime \prime} \Psi_{1}^{\prime}\right. & \left.+\left(\alpha+X^{\prime}\right)^{2} \Psi_{1}^{\prime \prime}\right\} \\
+\frac{1}{\pi^{\frac{1}{2}}} \int_{-\infty}^{-\gamma}\left\{X^{\prime \prime} \phi_{2}^{\prime}\right. & \left.+\left(\alpha+X^{\prime}\right)^{2} \phi_{2}^{\prime \prime}\right\} \exp \left(-\alpha^{2}\right) d \alpha \\
& +\frac{1}{\pi^{\frac{1}{2}}} \gamma^{\prime}\left(-\gamma+X^{\prime}\right) \exp \left(-\gamma^{2}\right)\left\{\Psi_{1}^{\prime}(0)-\phi_{2}^{\prime}(0)\right\},
\end{aligned}
$$




$$
\begin{gathered}
\theta Q^{\prime \prime}+2 Q^{\prime}-2 k \rho L X^{\prime \prime \prime}=\frac{-2 K}{\pi^{\frac{1}{2}}}\left[\int_{-\gamma}^{\infty}\left\{\alpha X^{\prime \prime} \Psi_{1}^{\prime}+\alpha\left(\alpha+X^{\prime}\right)^{2} \Psi_{1}^{\prime \prime}\right\} \exp \left(-\alpha^{2}\right) d \alpha\right. \\
+\int_{-\infty}^{-\gamma}\left\{\alpha X^{\prime \prime} \phi_{2}^{\prime}+\alpha\left(\alpha+X^{\prime}\right)^{2} \phi_{2}^{\prime \prime}\right\} \exp \left(-\alpha^{2}\right) d \alpha \\
\left.-\gamma^{\prime} \gamma\left(-\gamma+X^{\prime}\right) \exp \left(-\gamma^{2}\right)\left\{\Psi_{1}^{\prime}(0)-\phi_{2}^{\prime}(0)\right\}\right] .
\end{gathered}
$$

At $\theta=0$ these are

$$
\begin{gathered}
0=X_{2}\left(\Psi_{11}+\phi_{21}\right)+\frac{1}{2}\left(\Psi_{12}+\phi_{22}\right), \\
2 Q_{1}-12 k \rho L X_{3}=\frac{-2 K}{\pi^{\frac{1}{2}}}\left[X_{2}\left(\Psi_{11}-\phi_{21}\right)+\left(\Psi_{12}-\phi_{22}\right)\right] .
\end{gathered}
$$

Therefore

$$
\begin{gathered}
\phi_{22}=-\left(4 X_{2} \Psi_{11}+\Psi_{12}\right) \\
X_{3}=\frac{\pi^{\frac{1}{2}} Q_{1}+2 K \Psi_{12}}{6 \pi^{\frac{1}{2}} k \rho L}+\frac{K^{2} \Psi_{11}^{2}+K Q_{0}}{6 \pi^{\frac{1}{2}}(k \rho L)^{2}} .
\end{gathered}
$$

The second term is zero if $X_{2}=0$.

Further equations and coefficients are derived in the same manner. In Appendix A the first six equations are given.

To obtain the general equations for the unknown coefficients (4.6) and (4.8) are written as

$$
\begin{aligned}
T_{m}=\frac{1}{2} \sum_{n=0}^{\infty} n ! \theta^{n}\left[\frac{2}{\pi^{\frac{1}{2}}} \Psi_{1 n} \int_{-\gamma}^{\infty} \exp \left(-\alpha^{2}\right)(\alpha+\gamma)^{n} d \alpha\right. \\
\left.\quad+(-1)^{n} \frac{2 \phi_{2 n}}{\pi^{\frac{1}{2}}} \int_{\gamma}^{\infty} \exp \left(-\alpha^{2}\right) \frac{(\alpha-\gamma)^{n}}{n !} d \alpha\right] \\
=\frac{1}{2} \sum_{n=0}^{\infty} n ! \theta^{n}\left[\Psi_{1 n} i^{n} \operatorname{erfc}(-\gamma)+(-1)^{n} \phi_{2 n} i^{n} \operatorname{erfc}(\gamma)\right] \quad \ldots \ldots \ldots \ldots \ldots \ldots(4.14)
\end{aligned}
$$

and

$$
\begin{aligned}
Q_{n} \theta^{n+1} & -2 k \rho L \sum_{n=0}^{\infty} X_{n+1} \theta^{n}=\frac{-2 K}{\pi^{\frac{1}{2}}}\left[\frac{1}{2}\left\{\Psi_{1}(0)-\phi_{2}(0)\right\}\right. \\
& \left.+\frac{\theta}{2}\left\{\int_{-\gamma}^{\infty} e^{-\alpha^{2}} \Psi_{1}^{\prime}(\alpha \theta+X) d \alpha+\int_{-\infty}^{-\gamma} e^{-\alpha^{2}} \phi_{2}^{\prime}(\alpha \theta+X) d \alpha\right\}\right] \\
& =\frac{-K \theta}{\pi^{\frac{1}{2}}} \sum_{n=1}^{\infty} n \theta^{n}\left[\Psi_{1 n} \int_{-\gamma}^{\infty} \exp \left(-\alpha^{2}\right)(\alpha+\gamma)^{n-1} d \alpha\right. \\
& \left.+(-1)^{n} \phi_{2 n} \int_{\gamma}^{\infty} \exp \left(-\alpha^{2}\right)(\alpha-\gamma)^{n-1} d \alpha\right] \\
& =\frac{-K}{2} \sum_{n=1}^{\infty} n ! \theta^{n}\left[\Psi_{1 n} i^{n-1} \operatorname{erfc}(-\gamma)+(-1)^{n-1} \phi_{2 n} i^{n-1} \operatorname{erfc}(\gamma)\right] .
\end{aligned}
$$


The $n$th derivatives of these general equations evaluated at $\theta=0$ are

$$
\begin{aligned}
& 0=\sum_{r=0}^{n}(r !)^{2}\left(\begin{array}{l}
n \\
r
\end{array}\right) \frac{\partial^{n-r}}{\partial \theta^{n-r}}\left[\Psi_{1,} i^{r} \operatorname{erfc}(-\gamma)+(-1)^{r} \phi_{2 r^{2}} \operatorname{erfc}(\gamma)\right]_{\theta}=0 \\
& n ! Q_{n-1}-2 k \rho L(n !) X_{n+1}=\frac{-K}{2} \sum_{r=1}^{n}(r !)^{2}\left(\begin{array}{l}
n \\
r
\end{array}\right) \frac{\partial^{n-r}}{\partial \theta^{n-r}}\left[\Psi_{1,} r^{r-1} \operatorname{erfc}(-\gamma)\right. \\
& \left.+(-1)^{r-1} \theta_{2,} i^{r-1} \operatorname{erfc}(\gamma)\right]_{\theta=0}
\end{aligned}
$$

The general equations for $\phi_{2 n}$ and $X_{n+1}$ are

$$
\begin{aligned}
& \phi_{2 n}=-(-1)^{n}\left[\Psi_{1 n}+\frac{2^{n} \Gamma\left(\frac{1}{2} n+1\right)}{(n !)^{2}}\left\{\sum _ { r = 0 } ^ { n - 1 } ( r ! ) ^ { 2 } ( \begin{array} { l } 
{ n } \\
{ r }
\end{array} ) \frac { \partial ^ { n - r } } { \partial \theta ^ { n - r } } \left[\Psi_{1 r} i^{r-1} \operatorname{erfc}(-\gamma)\right.\right.\right. \\
& \left.\left.\left.+(-1)^{r} \phi_{2 r} i^{r} \operatorname{erfc}(\gamma)\right]_{\theta}=0\right\}\right]
\end{aligned}
$$

$2 k \rho L X_{n+1}=Q_{n-1}+\frac{K}{2(n !)}, \sum_{r=1}^{n}(r !)^{2}\left(\begin{array}{l}n \\ r\end{array}\right) \frac{\partial^{n-r}}{\partial \theta^{n-r}}\left[\Psi_{1,} i^{r-1} \operatorname{erf}(-\gamma)\right.$

$$
\left.+(-1)^{r-1} \phi_{2 r} i^{r-1} \operatorname{erfc}(\gamma)\right]_{\theta=0}
$$

where $i^{n} \operatorname{erfc}(0)=\frac{1}{2^{n} \Gamma\left(\frac{1}{2} n+1\right)}$ has been used in (4.18).

\section{Finite and semi-finite slabs}

It may be seen from the previous section that the method used is applicable, without change, for both finite and semi-infinite slabs. Finite regions are treated merely by the choice of an appropriate initial temperature selected according to the principles outlined in Section 3. The type of problem solved in Section 3 will be referred to as the "Standard Form".

\section{Reduction of problems involving solid spheres to standard form}

Consider a solid sphere, initially of radius $a$, which has, at $t=0$, a temperature distribution $V_{0}(r)$ such that $V_{0}(a)=T_{m}$. A flux $Q(\theta)$ is applied so that melting begins. The melt is continuously removed and the radius of the sphere at any time is given by $r=a-R(\theta)$ where $0 \leqq R(\theta)<a$. In the most general case $Q(\theta)$ may also be dependent on the instantaneous radius of the sphere but here $Q(\theta)$ will be considered to be a known function of $\theta$.

$V(r, t)$ the temperature within the sphere, satisfies the equations

$$
\begin{array}{ll}
\frac{\partial V}{\partial t}=k\left(\frac{\partial^{2} V}{\partial r^{2}}+\frac{2}{r} \frac{\partial V}{\partial r}\right) & 0 \leqq r<a-R(\theta), t>0 \\
V(r, 0)=V_{0}(r) & 0 \leqq r<a \\
V\{a-R, t\}=T_{m} & \\
Q(\theta)-L \rho \frac{d R}{d t}=\frac{K \partial V V}{\partial r} & \text { at } r=a-R(\theta) .
\end{array}
$$


Putting $U=r V$ the equations for $U(r, t)$ are

$$
\begin{aligned}
& \frac{\partial U}{\partial t}=k \frac{\partial^{2} U}{\partial r^{2}} \quad 0 \leqq r<a-R(\theta), t>0 \\
& U(r, 0)=r V_{0}(r) \quad 0 \leqq r<a \\
& U\{a-R, t\}=(a-R) T_{m} \\
& Q(\theta)-L \rho \frac{d R}{d t}=K\left\{\frac{-T_{m}}{a-R}+\frac{1}{a-R} \frac{\partial U}{\partial r}\right\} \text { at } r=a-r(\theta) \\
& U(0, t)=0 .
\end{aligned}
$$

Although reduced to a problem of linear flow this is not the standard form since the moving boundary moves towards the origin, not away from it. The further substitution $r^{\prime}=a-r$ achieves the "standard form " since the equations become

$$
\begin{aligned}
& \frac{\partial W}{\partial t}\left(r^{\prime}, t\right)=k \frac{\partial^{2} W\left(r^{\prime}, t\right)}{\partial r^{\prime 2}} \quad 0 \leqq r^{\prime}<a, t>0 \\
& W\left(r^{\prime}, 0\right)=\left(a-r^{\prime}\right) V_{0}\left(a-r^{\prime}\right) \quad 0 \leqq r^{\prime}<a \\
& W(R, t)=(a-R) T_{m} \\
& Q(\theta)-L \rho \frac{d R}{d t}=K\left\{\frac{-T_{m}}{a-R}-\frac{1}{a-R} \frac{\partial W}{\partial r^{\prime}}\right\} \text { at } r^{\prime}=R(\theta)
\end{aligned}
$$

where $W(a-r, t)=U(r, t)$.

$$
W(a, t)=0
$$

The form of solution of $(6.10)$ satisfying $(6.11)$ is

$$
\begin{aligned}
W\left(r^{\prime}, t\right)= & \frac{1}{\pi^{\frac{1}{2}}} \int_{-\infty}^{\infty} \exp \left[\frac{-R^{2}\left(y-y^{\prime}\right)^{2}}{\theta^{2}}\right]\left\{\left(a-R y^{\prime}\right) V_{0}\left(a-R y^{\prime}\right) U\left(R y^{\prime}\right)\right. \\
& \left.\quad+\phi_{2}\left(R y^{\prime}\right) U\left(-R y^{\prime}\right)\right\} \frac{R}{\theta} d y^{\prime} \quad \ldots . .(6.15) \\
= & \frac{1}{\pi^{\frac{1}{2}}} \int_{-\infty}^{\infty} \exp \left[\frac{-R^{2}\left(y-y^{\prime}\right)^{2}}{\theta^{2}}\right]\left\{\Psi_{1}\left(R y^{\prime}\right) U\left(R y^{\prime}\right)+\phi_{2}\left(R y^{\prime}\right) U\left(-R y^{\prime}\right)\right\} \frac{R}{\theta} d y^{\prime}
\end{aligned}
$$

where

$$
\Psi_{1}(R y)=\sum_{n=0}^{\infty} \Psi_{1 n}(R y)^{n}=(a-R y) V_{0}(a-R y) .
$$

Equation (6.14) is satisfied if $\left(a-r^{\prime}\right) V_{0}\left(a-r^{\prime}\right)$ is an odd function of $\left(a-r^{\prime}\right)$, that is if $V_{0}(r)$ is an even function of $r$.

Equation (6.12) is

$$
\begin{aligned}
(a-R) T_{m}=\frac{1}{\pi^{\frac{1}{2}}} \int_{-R / \theta}^{\infty} \exp \left(-\alpha^{2}\right) & \Psi_{1}(\alpha \theta+R) d \alpha \\
& +\frac{1}{\pi^{\frac{1}{2}}} \int_{-\infty}^{-R / \theta} \exp \left(-\alpha^{2}\right) \phi_{2}(\alpha \theta+R) d \alpha
\end{aligned}
$$


which, evaluated at $\theta=0$, yields

$$
2 a T_{m}=a T_{m}\left(1+\operatorname{erf} R_{1}\right)+\phi_{20}\left(1-\operatorname{erf} R_{1}\right)
$$

and therefore

$$
\phi_{20}=0 \text {. }
$$

Equation (6.13) is

$$
\begin{aligned}
(a-R)\left\{\theta Q(\theta)-2 L \rho k \frac{d R}{d \theta}\right\}+ & K T_{m} \theta=\frac{-2 K}{\pi^{\frac{1}{2}}}\left[\int_{-R / \theta}^{\infty} \alpha \exp (-\alpha)^{2} \Psi_{1}(\alpha \theta+R) d \alpha\right. \\
+ & \left.\int_{-\infty}^{-R / \theta} \alpha \exp \left(-\alpha^{2}\right) \phi_{2}(\alpha \theta+R) d \alpha\right] \ldots \ldots(6.19)
\end{aligned}
$$

which, at $\theta=0$, yields

Therefore

$$
-2 a L \rho k R_{1}=\frac{-K}{\pi^{\frac{1}{2}}}\left(\Psi_{10}-\phi_{20}\right)=0 .
$$

$$
R_{1}=0 .
$$

Two more differentiations result in

(which is zero if $\Psi_{11} \neq 0$ )

$$
\begin{gathered}
\phi_{21}=\Psi_{11} \\
R_{2}=\frac{K \Psi_{11}+K T_{m}+a Q_{0}}{4 a \rho k L}
\end{gathered}
$$

$$
\begin{gathered}
\phi_{22}=-\Psi_{12}-4 R_{2}\left(\Psi_{11}+T_{m}\right) \\
R_{3}=\frac{4 R_{2} K\left(\Psi_{11}+T_{m}\right)+\pi^{\frac{1}{2}} a Q_{0}}{6 a \rho k L} .
\end{gathered}
$$

To obtain the general equations (6.17) is written

$$
a T_{m}-T_{m} \sum_{n=1}^{\infty} R_{n} \theta^{n}=\frac{1}{2} \sum_{n=0}^{\infty} n ! \theta^{n}\left[\Psi_{1 n} i^{n} \operatorname{erfc}(-R / \theta)+(-1)^{n} \phi_{2 n} i^{n} \operatorname{erfc}(R / \theta)\right] ;
$$

the $n$th derivative with respect to $\theta$, evaluated at $\theta=0$, being

$$
\begin{aligned}
-n ! T_{m} R_{n}=\frac{1}{2} \sum_{r=0}^{n}(r !)^{2}\left(\begin{array}{l}
n \\
r
\end{array}\right) \frac{\partial^{n-r}}{\partial \theta^{n-r}}\left[\Psi_{1} r^{r} \operatorname{erfc}(-R / \theta)\right. \\
\left.+(-1)^{r} \phi_{2 r} i^{r} \operatorname{erfc}(R / \theta)\right]_{\theta}=0 .
\end{aligned}
$$

Therefore

$$
\begin{aligned}
& \phi_{2 n}=-(-1)^{n} \Psi_{1 n}-(-1)^{n} 2^{n} \Gamma\left(\frac{1}{2} n+1\right)\left\{2(n !) T_{m} R_{n}\right. \\
&+\left.\sum_{r=0}^{n-1}(r !)^{2}\left(\begin{array}{l}
n \\
r
\end{array}\right) \frac{\partial^{n-r}}{\partial \theta^{n-r}}\left[\Psi_{1 r^{r}} i^{r} \operatorname{erfc}(-R / \theta)+(-1)^{r} \phi_{2 r} i^{r} \operatorname{erfc}(R / \theta)\right]_{\theta}=0\right\} \\
& n \geqq 1 . \ldots . .(6.26)
\end{aligned}
$$


Equation (6.19) is written

$$
\begin{aligned}
K T_{m} \theta & +a \sum_{n=0}^{\infty} Q_{n} \theta^{n+1}-\left(\sum_{n=1}^{\infty} R_{n} \theta^{n}\right)\left(\sum_{n=0}^{\infty} Q_{n} \theta^{n+1}\right)-2 a \rho k L \sum_{n=1}^{\infty} n R_{n} \theta^{n-1} \\
& +2 \rho k L\left(\sum_{n=1}^{\infty} R_{n} \theta^{n}\right)\left(\sum_{n=1}^{\infty} n R_{n} \theta^{n-1}\right)=\frac{K}{2} \sum_{n=1}^{\infty} n ! \theta^{n}\left[\Psi_{1 n} i^{n-1} \operatorname{erfc}(-R / \theta)\right. \\
& \left.+(-1)^{n-1} i^{n-1} \operatorname{erfc}(R / \theta]\right)
\end{aligned}
$$

the $n$th derivative of which, evaluated at $\theta=0$, is

$$
\begin{aligned}
& n ! a Q_{n-1}-(n !) \sum_{r=1}^{n} R_{n-r} Q_{r-1}-2(n+1) ! R_{n+1}+2(n !) a k \rho L \sum_{r=1}^{n-1}(r+1) R_{n-r} R_{r+1} \\
& \quad=\frac{K}{2} \sum_{r=1}^{n}(r !)^{2}\left(\begin{array}{l}
n \\
r
\end{array}\right) \frac{\partial^{n-r}}{\partial \theta^{n-r}}\left[\Psi_{1 r} i^{r-1} \operatorname{erfc}(-R / \theta)+(-1)^{r-1} i^{r-1} \operatorname{erfc}(R / \theta)\right]_{\theta=0} .
\end{aligned}
$$

Therefore

$$
\begin{gathered}
2(n+1) !\left[a k \rho L R_{1}-1\right] R_{n+1}=n ! \sum_{r=1}^{n-1} R_{n-r} Q_{r-1}-n ! a Q_{n-1}-2(n !) a k \rho L \\
\times \sum_{r=1}^{n-2}(r+1) R_{n-r} R_{r+1} \\
+\frac{K}{2} \sum_{r=1}^{n}(r !)^{2}\left(\begin{array}{l}
n \\
r
\end{array}\right) \frac{\partial^{n-r}}{\partial \theta^{n-r}}\left[\Psi_{1 r} i^{r-1} \operatorname{erfc}(-R / \theta)+(-1)^{r-1} i^{r-1} \operatorname{erfc}(R / \theta)\right]_{\theta=0} \\
n \geqq 1 . \quad \ldots \ldots(6.6)
\end{gathered}
$$

Equations (6.26) and (6.29) are the general equations for the coefficients $\phi_{2 n}$ and $R_{n+1}$. Once these have been found the expression for the temperature distribution within the sphere is

$$
\begin{aligned}
u(r, t)= & \frac{1}{r \pi^{\frac{2}{2}}} \int_{-\infty}^{\infty} \exp \left[\frac{-R^{2}\left(y-y^{\prime}\right)^{2}}{\theta^{2}}\right]\left\{\Psi_{1}\left(a-R y^{\prime}\right) U\left(a-R y^{\prime}\right)\right. \\
& \left.+\phi_{2}\left(a-R y^{\prime}\right) U(-a-R y)\right\} \frac{R}{\theta} d y^{\prime} \\
= & \frac{-1}{(a-R y) \pi^{\frac{1}{2}}} \int_{(a-R y) / \theta}^{\infty} \exp \left(-\alpha^{2}\right) \Psi_{1}\{-\alpha \theta+(a-R y)\} d \alpha
\end{aligned}
$$

An application of this solution would be to find the time taken for a meteor to be consumed as it passes through the earth's atmosphere or to calculate the mass of material lost by a meteorite before striking the earth's surface.

E.M.S.--I 


\section{Reduction of problems involving spherical shells to standard form}

Consider a hollow spherical shell, initially comprising the region $b<r<a$, which has at $t=0$ a temperature distribution $V_{0}(r)$ such that $V_{0}(a)=T_{m}$. A flux $Q(\theta)$ is applied at $r=a$ so that melting begins. The melt is continuously removed and the outer surface of the shell at any time is given by $a-R(\theta)$ as long as $a-R(\theta)>b$. As in the previous section $Q(\theta)$ is a known function of $\theta$ and does not depend on $R(\theta)$.

If $V(r, t)$ is the temperature distribution within the shell then the equations for $V$ are

$$
\begin{aligned}
& \frac{\partial V}{\partial t}=k\left(\frac{\partial^{2} V}{\partial r^{2}}+\frac{2}{r} \frac{\partial V}{\partial r}\right) \quad b<r<a-R, t>0 \\
& V(r, 0)=V_{0}(r) \quad b<r<a \\
& V(a-R, t\}=T_{m} \\
& Q(\theta)-L \rho \frac{d R}{d t}=K \frac{\partial V}{\partial r} \quad \text { at } r=a-R(\theta) \\
& H V+K \frac{\partial V}{\partial r}=0 \quad \text { at } r=b .
\end{aligned}
$$

Substituting $U=r V$ these equations become

$$
\begin{aligned}
& \frac{\partial U}{\partial t}=k \frac{\partial^{2} U}{\partial r^{2}} \quad b<r<a-R, t>0 \\
& U(r, 0)=r V_{0}(r) \quad b<r<a \\
& U(a-R, t)=(a-R) T_{m} \\
& Q(\theta)-L \rho \frac{d R}{d t}=K\left\{\frac{-T_{m}}{a-R}+\frac{1}{a-R} \frac{\partial U}{\partial r}\right\} \text { at } r=a-R \\
& \frac{H U}{b}-K\left\{\frac{-1}{b^{2}} U+\frac{1}{b} \frac{\partial U}{\partial r}\right\}=0 \quad \text { at } r=b .
\end{aligned}
$$

The further substitution $r^{\prime}=a-r$ transforms the equations into the standard form since the problem then becomes one of ablation in a slab $0<R(\theta)<r<a-b$. If $W(a-r, t)=U(r, t)$ equations (7.6)-(7.10) become

$$
\begin{array}{ll}
\frac{\partial W\left(r^{\prime}, t\right)}{\partial t}=k \frac{\partial^{2} W\left(r^{\prime}, t\right)}{\partial r^{2}} & 0<r^{\prime}<a-b, t>0 \\
W\left(r^{\prime}, 0\right)=\left(a-r^{\prime}\right) V_{0}\left(a-r^{\prime}\right) & 0<r^{\prime}<a-b \\
W(R, t)=(a-R) T_{m} & \ldots \ldots \ldots \ldots \ldots \ldots \ldots \ldots \ldots \ldots \ldots \\
Q(\theta)-L \rho \frac{d R}{d t}=K\left\{\frac{-T_{m}}{a-R}-\frac{1}{a-R} \frac{\partial W}{a r^{\prime}}\right\} & \text { at } r^{\prime}=R \ldots \ldots \ldots \ldots \\
\frac{H}{b} W-K\left\{\frac{-1}{b^{2}} W-\frac{1}{b} \frac{\partial W}{\partial r^{\prime}}\right\}=0 & \text { at } r^{\prime}=a-b . \ldots \ldots
\end{array}
$$


The problem can be solved if a suitable initial temperature can be found. Thus if $H=0, W\left(r^{\prime}, 0\right)$ must be expressible as an odd function of $r^{\prime}-(a-b)$. This means that $r V_{0}(r)$ must be an odd function of $r-b$.

The rest of the solution is identical with the work of Section 6 . The only difference arises from the necessity of condition (6.14) for a solid sphere whereas (7.15) allows a variety of situations at the inner surface of a hollow shell.

\section{Cooling of molten regions by radiation}

By using Portnov's method it is possible to find the temperature distribution in both phases of a slab, initially liquid, cooling by radiation into a medium at zero temperature so that a solid phase advances into the region $x>0$ from the face $x=0$. It is sufficient to say here that two temperature distributions are postulated and that the differentiations, etc., are carried out as above. The algebra is, however, rather more cumbersome since sets of four simultaneous equations have to be solved for four unknown coefficients. The first few coefficients have been worked out and agree with those given in (2).

An important application would be to the cooling of spheres; for example the cooling of the earth could be investigated. Preliminary work shows that it is quite feasible to find short-time solutions for the case of a sphere, cooling at the outer surface by radiation, with heat generation at a rate $A_{0} \exp [b(a-r)]$ where $a$ is the radius of the sphere and $A_{0}$ and $b$ are positive constants. This would be a realistic model to use in investigating the cooling of a molten earth if heating by radioactive decay within the mantle is to be taken into account.

\section{Some worked examples}

The first example is one for which the first few terms have already been worked out by Boley (2) using a method based on Duhamel's theorem.

A semi-infinite solid $x>0$, at zero temperature when $t=-t_{m}$, constant flux $Q_{0}$ into the slab at $x=0$ for $t>-t_{m}$.

At $t=0$ the temperature is

$$
V_{0}(x)=\frac{2 Q_{0}}{K}\left(k t_{m}\right)^{\frac{1}{2}} \operatorname{erfc}\left(x / 2\left(k t_{m}\right)^{\frac{1}{2}}\right)
$$

where $t_{m}$ has to found from

$$
\begin{aligned}
T_{m} & =\frac{2 Q_{0}}{K}\left(k t_{m}\right)^{\frac{1}{2}} \operatorname{erfc}(0) \\
& =\frac{2 Q_{0}}{K}\left(k t_{m} / \pi\right)^{\frac{1}{2}} .
\end{aligned}
$$

Therefore

$$
t_{m}=\pi K^{2} T_{m}^{2} / 4 k Q_{0}^{2} .
$$

In this case $\Psi_{1}(x)=V_{0}(x)$, that is

$$
\Psi_{1}(x)=\frac{2 Q_{0}}{K}\left[\left(k t_{m} / \pi\right)^{\frac{4}{2}} \exp \left(-x^{2} / 4 k t_{m}\right)-\frac{x}{2} \operatorname{erfc}\left(x / 2\left(k t_{m}\right)^{\frac{1}{2}}\right)\right]
$$


whence

$$
\begin{array}{ll}
\Psi_{10}=\frac{2 Q_{0}}{K}\left(k t_{w} j \pi\right)^{\frac{1}{2}}=T_{m}, & \Psi_{11}=-Q_{0} / K \\
\Psi_{12}=Q_{0}^{2} / \pi K^{2} T_{m}, & \Psi_{13}=0 \\
\Psi_{14}=-Q_{0}^{4} / 6 \pi^{2} K^{4} T_{m}^{3} & \Psi_{15}=0
\end{array}
$$

It is found that

$$
\begin{aligned}
\phi_{20}= & T_{m} \quad \phi_{21}=-Q_{0} / K \\
\phi_{22}= & Q_{0}^{2} / \pi K^{2} T_{m} \quad \phi_{23}=-2 Q_{0}^{3} / 3 \pi k \rho L T_{m} K^{2} \\
\phi_{24}= & -Q_{0}^{4}\left[\left\{6 \pi(k \rho L)^{2} T_{m} K^{2}\right\}^{-1}-16\left\{9 \pi^{3} k \rho L T_{m}^{2} K^{3}\right\}^{-1}-\left\{6 \pi^{2} T_{m}^{3} K^{4}\right\}^{-1}\right] \quad(9.6) \\
\phi_{25}= & Q_{0}^{5}\left[\left\{15 \pi^{2}(k \rho L) T_{m}^{3} K^{4}\right\}^{-1}-16\left\{45 \pi^{3}(k \rho L)^{2} T_{m}^{2} K^{3}\right\}^{-1}\right. \\
& \left.\quad-\left\{30 \pi(k \rho L)^{3} T_{m} K^{2}\right\}^{-1}-\left\{8 \pi^{2}(k \rho L)^{2} T_{m}^{2} K^{3}\right\}^{-1}+\left\{6 \pi^{2}(k \rho L)^{2} T_{m}^{2} K^{3}\right\}^{-1}\right] \\
X_{1}= & 0, X_{2}=0, X_{3}=Q_{0}^{2} /\left(3 \pi^{3 / 2} k \rho L T_{m} K\right) \\
X_{4}= & -Q_{0}^{3} /\left\{16 \pi(k \rho L)^{2} T_{m} K\right\} \\
X_{5}= & Q_{0}^{4}\left[\frac{16}{45 \pi^{7 / 2}(k \rho L)^{2} T_{m}^{2} K^{2}}+\frac{1}{30 \pi^{3 / 2}(k \rho L)^{3} T_{m} K}-\frac{1}{15 \pi^{5 / 2}(k \rho L) T_{m}^{2} K^{2}}\right] \quad(9.7) \\
X_{6}= & Q_{0}^{5}\left[\left\{96 \pi^{2}(k \rho L)^{2} T_{m}^{3} K^{3}\right\}^{-1}-\left\{192 \pi(k \rho L)^{4} T_{m} K\right\}^{-1}\right.
\end{aligned}
$$

As a second example the case of a semi-infinite region exposed to a pulse of heat is discussed.

In this example the semi-infinite region $x>0$ is considered to be at zero temperature $a t=-t_{m}$, at which time a flux of heat $Q(t)=r \pi^{-\frac{1}{2}} \exp \left(-r^{2} t^{2}\right)$, in suitable units, is applied to the face $x=0$. For large values of $r$ this expression will be a good approximation to an instantaneous unit, pulse at $t=0$ since $t_{m}$ will be very small.

By direct application of Duhamel's theorem the temperature for $-t_{m}<t<0$ is found to be

$$
\frac{r}{\pi K} k^{\frac{1}{2}} \int_{-t_{m}}^{t+t_{m}} \exp \left[-r^{2}\left(t+t_{m}-\tau\right)^{2}\right] \exp \left[-x^{2} / 4 k\left(t+t_{m}\right)\right] \frac{d \tau}{\tau^{\frac{1}{2}}} .
$$

When $t=0$ this expression becomes

$$
\begin{aligned}
V_{0}(x) & =\frac{r}{\pi K} k^{\frac{1}{2}} \exp \left[-x^{2} / 4 k t_{m}\right] \int_{0}^{t_{m}} \exp \left[-r^{2}\left(t_{m}-\tau\right)^{2} \frac{d \tau}{\tau^{\frac{1}{2}}}\right. \\
& =\frac{r}{\pi K} k^{\frac{1}{2}} \exp \left[-x^{2} / 4 k t_{m}\right]\left[2 t_{m}^{\frac{1}{2}}+O\left(t_{m}^{3 / 2}\right)\right] .
\end{aligned}
$$


If $r$ is large $t_{m}$ will be small and $O\left(t_{m}^{3 / 2}\right)$ may be ignored so that a good approximation will be

$$
V_{0}(x)=\frac{2 r\left(k t_{m}\right)^{\frac{1}{2}}}{\pi K} \exp \left[-x^{2} / 4 k t_{m}\right]
$$

Provided that $Q(t)$ is large enough to cause melting to begin when $t=0$ the value of $t_{m}$ may be found from

$$
T_{m}=\frac{2 r\left(k t_{m}\right)^{\frac{1}{2}}}{\pi K}
$$

i.e.

$$
t_{m}=\frac{\pi^{2} K^{2} T_{m}^{2}}{4 r^{2} k}
$$

Using this value of $t_{m}$ in (9.8)

$$
V_{0}(x)=T_{m} \exp \left[-r^{2} x^{2} / \pi^{2} K^{2} T_{m}^{2}\right] .
$$

From this result the coefficients of $\phi_{1}(x)$ are found to be

$$
\begin{aligned}
\phi_{1,2 n} & =\frac{(-1)^{n} r^{2 n}}{n ! \pi^{2 n} K^{2 n} T_{m}^{2 n-1}} \quad n=0,1,2,3, \ldots \\
\phi_{1,2 n+1} & =0
\end{aligned}
$$

Equation (9.10) also shows that $V_{0}(x)$ is very small when $r$ is large, except at $x=0$ where its value is $T_{m}$.

The series expansion for $Q(t)$ in powers of $\theta$ is found from

therefore

$$
\begin{gathered}
Q(\theta)=r \pi^{-\frac{1}{2}} \exp \left[-r^{2} \theta^{4} / 16 k^{2}\right] \\
=r \pi^{-\frac{1}{2}} \sum_{n=0}^{\infty} \frac{(-1)^{n}}{n !}\left(\frac{r^{2} \theta^{4}}{16 k^{2}}\right)^{n}
\end{gathered}
$$

$$
Q_{n}= \begin{cases}\frac{(-1)^{n} r^{2 n+1}}{\pi^{\frac{1}{2}} n ! 16^{n} k^{2 n}} & n=0,4,8,12, \ldots \\ 0 & n=1,2,3,5,6,7,9, \ldots\end{cases}
$$

Using (9.11), (9.12) and the results of Section 4 it is found that

$$
\begin{gathered}
\phi_{20}=T_{m}, \phi_{21}=0, \phi_{22}=\frac{-r^{4}}{\pi^{4} K^{4} T_{m}^{3}} \quad \cdots \\
X_{1}=0, X_{2}=\frac{r}{4 \pi^{\frac{1}{2}} k \rho L}, X_{3}=\frac{r^{2}}{3 \pi^{5 / 2} k \rho L K T_{m}} .
\end{gathered}
$$

Thus a good approximation to $X(t)$ is

$$
X(t)=\frac{r}{\pi^{\frac{1}{2}} \rho L} t-\frac{8 k^{2} r^{2}}{3 \pi^{5 / 2} \rho L K T_{m}} t^{3 / 2}
$$




$$
\text { Now } \frac{d X}{d t}=0 \text { when } t=\frac{\pi^{4} K^{2} T_{m}^{2}}{16 r^{2} k^{4}}=t_{f}, \text { say. }
$$

Hence the melting ceases after a layer with thickness

$$
\begin{aligned}
& X\left(t_{f}\right)=\frac{\pi^{7 / 2} K^{2} T_{m}^{2}}{16 r \rho L k^{4}}-\frac{\pi^{7 / 2} K^{2} T_{m}^{2}}{18 r \rho L k^{4}} \\
& =\frac{\pi^{7 / 2} K^{2} T_{m}^{2}}{144 r \rho L k^{4}} \\
& =\frac{r t_{f}}{9 \pi^{\frac{2}{2}} \rho L} .
\end{aligned}
$$

has been removed.

As a last example the ablation of a finite slab, $0<x<a$, perfectly insulated at $x=0$ has been chosen.

Let the slab $0<x<a$ be at zero temperature when $t=-t_{m}$. For $t>-t_{m}$ a constant flux $Q_{0}$ flows into the region and at $t=0$ the face $x=0$ is at temperature $T_{m}$ so that melting begins. The face $x=a$ is perfectly insulated so that no heat can pass through it.

The initial temperature is given by Example $\mathbf{A}$ of Section 3. Rearranged in powers of $x$ the trigonometric series becomes the absolutely convergent series

$$
\begin{aligned}
& \phi_{0}(x)=Q_{0}\left(\frac{t_{m}}{\rho c a}+\frac{a}{3 K}-\frac{2 a S_{0}}{K \pi^{2}}\right)-\frac{Q_{0}}{K} x+\frac{Q_{0}}{K}\left(S_{1}-\frac{1}{2 a}\right) x^{2}- \\
& \text {. . . . . }-(-1)^{n} \frac{2 Q_{0} \pi^{2 n-2} S_{n}}{K a^{2 n-1}(2 n) !} x^{2 n}
\end{aligned}
$$

where

$$
S_{n}=\sum_{r=1}^{\infty} r^{2 n-2} \exp \left[-r^{2} k \pi^{2} t_{m} / a^{2}\right]
$$

From $\phi_{1}(0)=T_{m}$ it is found that

$$
t_{m}=\rho c a\left[\frac{T_{m}}{Q_{0}}-\frac{a}{3 K}+\frac{2 a S_{0}}{K \pi^{2}}\right] .
$$

If $t_{m}$ is small the series representation for $S_{0}$ given by (9.19) will be slowly convergent and it will then be useful to use the alternative expression for $V_{0}(a-x)$ given in Example $\mathbf{A}$.

Using the coefficients $\phi_{1 n}$ given in (9.18) it is found that

$$
\begin{aligned}
& \phi_{20}=T_{m}, \phi_{21}=\frac{-Q_{0}}{K}, \phi_{22}=-Q_{0} \frac{\left(2 a S_{1}-1\right)}{2 K a} \\
& \phi_{23}=\frac{-Q_{0}^{2}\left(2 a S_{1}-1\right)}{6 a k \rho L K}
\end{aligned}
$$




$$
\begin{gathered}
\phi_{24}=\frac{\pi^{2} Q_{0} S_{2}}{12 K a^{3}}-\frac{2 Q_{0}^{2}\left(2 a S_{1}-1\right)^{2}}{9 \pi K a^{2}(k \rho L)}-\frac{Q_{0}^{3}\left(2 a S_{1}-1\right)}{24 K a(k \rho L)^{2}} \\
\phi_{25}=\frac{\pi^{2} Q_{0}^{2} S_{2}}{30 K a^{3}(k \rho L)}-\frac{Q_{0}^{3}\left(2 a S_{1}-1\right)}{45 \pi K a^{2}(k \rho L)^{2}}-\frac{Q_{0}^{4}\left(2 a S_{1}-1\right)}{120 K a(k \rho L)^{3}}-\frac{Q_{0}^{3}\left(2 a S_{1}-1\right)^{2}}{192 K a^{2}(k \rho L)^{2}} \\
X_{1}=0, X_{2}=0, X_{3}=\frac{Q_{0}\left(2 a S_{1}-1\right)}{12 \pi^{\frac{1}{2}} a k \rho L} \\
X_{4}=\frac{-Q_{0}^{2}\left(2 a S_{1}-1\right)}{64 a(k \rho L)^{2}} \\
X_{5}=\frac{Q_{0}^{3}\left(2 a S_{1}-1\right)}{120 \pi^{\frac{1}{3}} a(k \rho L)^{3}}+\frac{2 Q_{0}^{2}\left(2 a S_{1}-1\right)}{45 \pi^{3 / 2} a^{2}(k \rho L)^{2}}-\frac{Q_{0} \pi^{3 / 2} S_{2}}{30 a^{3} k \rho L} \\
X_{6}=\frac{1}{192}\left[\frac{19 Q_{0}^{3}\left(2 a S_{1}-1\right)^{2}}{32 \pi a^{2}(k \rho L)^{3}}-\frac{Q_{0}^{4}\left(2 a S_{1}-1\right)^{3}}{24 \pi a^{3}(k \rho L)^{4}}-\frac{3 Q_{0}^{3}\left(2 a S_{1}-1\right)}{4 \pi a^{2}(k \rho L)^{3}}-\frac{Q_{0}^{4}\left(2 a S_{1}-1\right)}{4 a(k \rho L)^{4}}\right. \\
\left.+\frac{\pi^{2} Q_{0}^{2} S_{2}}{a^{3}(k \rho L)^{2}}\right] .
\end{gathered}
$$

\section{Conclusion}

It has been shown that Portnov's method provides a powerful means of investigating many important problems of heat conduction where phase changes occur. It is easily seen that the method is applicable to any situation where the appropriate heat equation may be transformed into the case of linear flow, for example the thin rod with radiation from its surface. By combining step functions with additional "fictitious" temperature distributions cases involving more than one moving boundary may be investigated and also slabs with time dependent fluxes or temperatures at the fixed boundary.

\section{Appendix}

The first six sets of equations found from equations (4.6) and (4.8) when $X_{1}=\mathrm{X}_{2}=0$.

1. (a) $2 T_{m}=\Psi_{10}\left(1+\operatorname{erf} X_{1}\right)+\phi_{20}\left(1-\operatorname{erf} X_{1}\right)$

(b) $\pi^{\frac{1}{2}} 2 k \rho L X_{1}=K\left(\Psi_{10}-\phi_{20}\right)$

2. (a) $0=\Psi_{11}-\phi_{21}$

(b) $2 Q_{0}-8 k \rho L X_{2}=-K\left(\Psi_{11}+\phi_{21}\right)$

3. (a) $0=\Psi_{12}+\phi_{22}$

(b) $\pi^{\frac{1}{2}}\left(Q_{1}-6 k \rho L X_{3}\right)=-K\left(\Psi_{12}-\phi_{22}\right)$

4. (a) $0=\pi^{\frac{1}{2}} X_{3}\left(\Psi_{11}+\phi_{21}\right)+\left(\Psi_{13}-\phi_{23}\right)$

(b) $4\left(Q_{2}-8 k \rho L X_{4}\right)=-3 K\left(\Psi_{13}+\phi_{23}\right)$ 
5. (a) $0=4 \pi^{\frac{1}{2}} X_{4}\left(\Psi_{11}+\phi_{21}\right)+8 X_{3}\left(\Psi_{12}-\phi_{22}\right)$

$$
+3 \pi^{\frac{1}{2}}\left(\Psi_{14}+\phi_{24}\right)
$$

(b) $4 \pi^{\frac{1}{2}}\left(Q_{3}-10 k \rho L X_{5}\right)=-K\left[4 \pi^{\frac{1}{2}} X_{3}\left(\Psi_{12}+\phi_{22}\right)+12 X_{2}\left(\Psi_{12}+\phi_{22}\right)\right.$

$$
\left.+8\left(\Psi_{14}-\phi_{24}\right)\right]
$$

6. (a) $0=2 \pi^{\frac{1}{2}} X_{5}\left(\Psi_{11}+\phi_{21}\right)+4 X_{4}\left(\Psi_{12}-\phi_{22}\right)$

$$
+3 \pi^{\frac{1}{2}} X_{3}\left(\Psi_{13}+\phi_{23}\right)+4\left(\Psi_{15}-\phi_{25}\right)
$$

(b) $\pi^{\frac{1}{2}}\left(Q_{4}-12 k \rho L X_{6}\right)=-K\left[\pi^{\frac{1}{2}} X_{4}\left(\Psi_{12}+\phi_{22}\right)\right.$

$$
\left.+3 X_{3}\left(\Psi_{13}-\phi_{23}\right)+\frac{15}{8} \pi^{\frac{1}{2}}\left(\Psi_{15}+\phi_{25}\right)\right]
$$

\section{REFERENCES}

(1) I. G. PORTNOv, Exact solution of freezing problem with arbitrary temperature variation on fixed boundary, Soviet Physics, Doklady, 7 (1962), pp. 186-189.

(2) B. A. BoLEY, A method of heat conduction analysis of melting and solidification problems, J. Math. Phys., 40 (1961), pp. 300-313.

(3) H. G. Landau, Heat conduction in a melting slab, Quart. of Appl. Math., 8 (1950), pp. 81-94.

(4) J. I. Masters, Problem of intense surface heating of a slab accompanied by a change of phase, J. Appl. Phys., 27 (1956), pp. 477-485.

(5) H. S. CARSLAW and J. C. JAEGER, Conduction of Heat in Solids (Oxford University Press, London, 1957).

Nova Scotia Technical College

HALIFAX

Canada 